\title{
Impacto de una intervención dirigida a incrementar el conocimiento de la enfermedad renal sobre el inicio oportuno de la terapia sustitutiva
}

\author{
Emeterio Burgos Jiménez ${ }^{1}$ - Manuela A. Meléndez Balderrama² - Eduwiges Meza Coronado² - Karla G. Agramón Cota² - \\ María C. Pereyra Hernández² - Norma L. Martínez Menchaca²
}

${ }^{1}$ Lic. En Enfermería, Departamento de educación e investigación

${ }^{2}$ Enfermera General, Curso Postécnico de Enfermería en Nefrología

Hospital general de Zona No 17, Instituto Mexicano del Seguro Social (IMSS). Monterrey; México

\section{Resumen}

Introducción: En México se estima que entre el 70 y el $80 \%$ de los pacientes que ingresan a diálisis 10 hacen de forma urgente, esto se traduce en mayor morbimortalidad, mayores costos y menor eficacia organizativa en los servicios de nefrología.

Objetivo: Evaluar el impacto de una intervención dirigida a incrementar el conocimiento de la enfermedad renal sobre el inicio oportuno de la terapia sustitutiva.

Hipótesis: El promedio del grupo en el conocimiento de la enfermedad renal después de la intervención es mayor al promedio previo a la misma.

Material y Métodos: Diseño pre experimental (antes - después) muestra de 65 pacientes en estadio 5 de la IR (IC 95\%) se sometió al grupo a cuatro sesiones educativas: Sobre la enfermedad renal crónica, la nutrición y la enfermedad renal crónica, cambios en el estilo de vida y diálisis peritoneal, lo que necesita saber. Se utilizo un cuestionario de 26 ítems (KR .8). Se utilizó estadística descriptiva y $\mathrm{t}$ de students (ns $>0.05$ ).

Resultados: Antes de la intervención el promedio de conocimiento fue de 60.76 incrementándose en 35.08 puntos posteriores a la misma. Previo a la intervención el nivel bueno se encuentra en $23.1 \%$ incrementándose a $98.5 \%$. Se lograron cambios significativos en el nivel de conocimiento $(t=13.207$ ns.000) El 93.80 aceptaron iniciar tratamiento posterior a la intervención.

Conclusiones: Proporcionar educación sobre la enfermedad renal y sus opciones de tratamiento incrementa el conocimiento de la misma, favoreciendo la toma de decisiones acertadas para el inicio oportuno de la terapia sustitutiva.

PALABRAS CLAVE:

- DIÁLISIS

- INSUFICIENCIA RENAL CRÓNICA

- INICIO OPORTUNO

- ESTRATEGIA EDUCATIVA 
Impact of an intervention aimed at increasing knowledge of kidney disease on the timely commencement of replacement therapy

\section{Abstract}

Introduction: In Mexico it is estimated that between 70 and $80 \%$ of patients who are admitted for dialysis are admitted as emergencies. This leads to higher mortality, higher costs and reduced organizational efficacy in the nephrology services.

Aim: To assess the impact of an intervention aimed at increasing knowledge of kidney disease on the timely commencement of replacement therapy.

Hypothesis: The group average knowledge of kidney disease after the intervention is higher than the average prior to the intervention.

Material and Methods: Pre-experimental design (before-after): a sample of 65 patients with stage 5 CKD (95\% CI) underwent four group educational sessions: About chronic kidney disease, Nutrition and chronic kidney disease, Changes in lifestyle and Peritoneal dialysis, what you need to know. A questionnaire containing 26 items (KR.8) was used. Descriptive statistics and the student's t-test were used (ns $>0.05$ ).

Results: Before the intervention, average knowledge was 60.76 which increased by 35.08 points after the intervention. Prior to the intervention, good level was at $23.1 \%$, and increased to $98.5 \%$. Significant changes in the level of knowledge were achieved $(t=$ 13.207 ns.000) $93.80 \%$ agreed to start treatment after the intervention.

Conclusions: Providing education on kidney disease and the treatment options increases knowledge of the disease, favouring correct decision-making for the timely commencement of replacement therapy.

\section{KEY WORDS:}

- DIALYSIS

- CHRONIC KIDNEY DISEASE

- TIMELY COMMENCEMENT

- EDUCATIONAL STRATEGY

\section{Introducción}

La insuficiencia renal crónica (IRC), representa un problema de salud pública de importancia creciente en México. Tan solo para el Instituto Mexicano del seguro Social (IMSS) las nefropatías constituyen la primera causa de ingresos hospitalarios con el $3.12 \%$ y la tercera causa de muerte hospitalaria con el $5 \%^{1}$.

El aumento en la incidencia de la patología renal esta condicionado, principalmente, por la diabetes mellitus y la hipertensión arterial. Se estima que de cada 100 pacientes diabéticos 14 desarrollaran nefropatía; lo que hoy nos lleva a tener 70,000 pacientes con tratamiento sustitutivo (diálisis y hemodiálisis) de los cuales 50,000 son atendidos dentro de nuestra institución, con todas las implicaciones clínicas, sociales y económicas que esto significal.

El inicio de la terapia de reemplazo es un punto crucial para el pronóstico con IRC ya que la supervivencia del paciente depende en gran parte, del estado del mismo en dicho momento; el inicio de diálisis no programada se ha asociado a mayor morbilidad, mortalidad y altos costos $^{2}$.

Las tendencias en el inicio de la diálisis varían de país a país e incluso de centro a centro. Mientras en España el $50 \%$ de los pacientes inician en condiciones de urgencias ${ }^{3}$, en México este porcentaje se aumenta hasta un $80 \%$, además ingresan con una función glomerular disminuida hasta de $3 \mathrm{ml} / \mathrm{min}$ en comparación a lo sugerido por la National Kydney Fundation de $10-15 \mathrm{ml} / \mathrm{min}^{4}$.

Esta misma fundación recomienda que todo aquel paciente que alcance el estadio cuatro de la enfermedad renal debe recibir educación programada sobre la función de los riñones y la enfermedad renal como tal, con la intención de que plantear el tratamiento de diálisis permite el inicio de la misma en un tiempo apropiado 5 .

El profesional de enfermería nefrológica, debe asegurarse de que el paciente en la etapa prediálisis disponga del adecuado cuidado enfermero y reciba la información básica sobre el tratamiento y el proceso de toma de decisiones, ya que preparar al paciente oportunamente para afrontar adecuadamente su enfermedad, puede evitar complicaciones y aminorar la ansiedad para la 
adaptación de la futura vida del paciente en la diálisis ${ }^{(5)}$. El objetivo del presente trabajo es evaluar el impacto de una intervención dirigida a incrementar el conocimiento de la enfermedad renal sobre el inicio oportuno de la terapia sustitutiva.

\section{Material y métodos}

Esta investigación se ha realizado a través de un estudio de intervención antes - después sin grupo de control con una duración de 6 meses. La población de estudio estuvo conformada por 78 pacientes mayores de edad diagnosticados con IRC en estadio 5 según la clasificación propuesta por la NKF valorada mediante filtrado glomerular los cuales fueron seleccionados de los pacientes que acudieron a la consulta de nefrología durante los meses de noviembre del 2010 a febrero del 2011. Se excluyeron a aquellos pacientes que no desearon participar en el estudio y se eliminaron a quienes tuvieron que iniciar diálisis de forma urgente, desertaron voluntariamente o fallecieron.

La muestra quedo constituida finalmente por 65 pacientes a quienes, previo consentimiento informado y con las debidas autorizaciones del comité local de investigación, se les aplico la intervención educativa consistente en cuatro sesiones con una hora de duración y un intervalo de 7 días entre ellas con los temas descritos en la tabla 1.

\begin{tabular}{|c|l|}
\hline No de sesión & \multicolumn{1}{c|}{ Tema } \\
\hline $\mathbf{1}$ & Pre test \\
& "Sobre la enfermedad renal crónica" \\
\hline $\mathbf{2}$ & "La nutrición y la enfermedad renal crónica" \\
\hline $\mathbf{3}$ & "Cambios en el estilo de vida" \\
\hline $\mathbf{4}$ & "Diálisis peritoneal, lo que necesita saber" \\
& Pos test \\
\hline
\end{tabular}

Tabla 1: Intervención educativa en el paciente de prediálisis dirigida al conocimiento de la enfermedad renal y su opción de tratamiento

Las variables independientes fueron medidas a través de un cuestionario formado por seis preguntas que valoraban los datos demográficos (edad, sexo, estado civil, escolaridad) y la situación de la enfermedad (etiología, comorbilidad). Para valorar el nivel de conocimiento de la enfermedad renal se construyo un cuestionario de 26 ítems con respuesta dicotómica si - no, el cual comprende los cuatro temas relacionados con el control del programa educativo al paciente en la etapa predialisis y que fueron expuestos durante la intervención. El nivel de conocimiento se califico como malo, regular y bueno (menos de 10 respuestas correctas, de 11 a 20 y de 21 a 26 respectivamente). El cuestionario se sometió a revisión de expertos y la consistencia interna fue de 8 (KR).

La información recogida en la investigación ha sido analizada mediante el programa estadístico SPSS. 18. Los resultados obtenidos siguieron una distribución normal (KS) por lo que utilizamos prueba de $t$ de Student para muestras relacionadas, y Chi-cuadrada para la asociación entre el nivel de conocimiento y la aceptación en iniciar tratamiento de diálisis. El nivel de significancia de este estudio fue $p$ valor $>$ 0.05 .

\section{Resultados}

La muestra del estudio estuvo formada por 65 pacientes predominando los pacientes mayores de 60 años con un $40 \%$. En cuanto al género hay una proporción mayor de mujeres con un porcentaje de $58.5 \%$ frente a un $41.5 \%$ de varones. En relación al estado civil el $73.8 \%$ de los pacientes están casados, el $10.8 \%$ divorciados, el $9.2 \%$ viudos, el $4.6 \%$ solteros y un $1.5 \%$ viven en unión libre. En cuanto a la escolaridad, el nivel educación primaria al igual que el nivel educación secundaria correspondieron a un $40 \%$ cada uno, seguido del nivel bachillerato con un $9.2 \%$ y el profesional con un $6.2 \%$ y $\sin$ escolaridad el $4.6 \%$.

En lo referente a la etiología de la IRC el $67 \%$ es secundaria a diabetes mellitus y el $32.3 \%$ a hipertensión arterial. La comorbilidad predominante fue la cardiovascular con un $69.2 \%$, seguida de enfermedades respiratorias con un $7.7 \%$ y un $23.1 \%$ de pacientes que no registra comorbilidad.

Los resultados en cuanto al nivel de aprovechamiento en el conocimiento de la enfermedad renal del total de pacientes, se reflejan en la tabla 2. 


\begin{tabular}{|l|l|l|l|l|}
\hline & Media & T & Ns \\
\hline Función del riñón y conocimiento de la enfermedad renal & 31.9 & 4.584 & 8 & .002 \\
\hline Estilo de vida & 47.6 & 7.585 & .002 \\
\hline Diálisis & 33.8 & 6.169 & .000 \\
\hline Dieta & 20.0 & 4.345 & .049 \\
\hline Promedio & 35.08 & 13.207 & 64 & .000 \\
\hline
\end{tabular}

Tabla 2: Comparación de medias en prueba pre test-pos test, $t$ de Student nivel de significancia 0.05

La decisión de aceptar iniciar el tratamiento sustitutivo en el momento del pos test en relación al nivel de conocimiento se muestra en la tabla 3.

\begin{tabular}{|l|c|c|c|}
\hline Inicio de la diálisis & Malo & Regular & Bueno \\
\hline Acepta & 0 & 0 & 61 \\
\hline No acepta & 0 & 1 & 3 \\
\hline
\end{tabular}

Tabla 3: Chi-cuadrada 15.488. Ns .000

\section{Discusión}

Las estrategias educativas han sido utilizadas para la mejora y aumento de los conocimientos en distintas patologías, favoreciendo los niveles de adherencia a los tratamientos específicos y correlacionándolas con indicadores clínicos. Encontrando niveles de conocimiento superiores significativos de la enfermedad renal posterior a una intervención ${ }^{6}$.

En nuestro hospital la población que cursa con IRC es de 922 pacientes de los cuales 302 ya se encuentran en alguna terapia sustitutiva, 620 cursan en algún estadio de la progresión de la enfermedad renal de los cuales 78 de ellos se encuentran en estadio 5, siendo este grupo quien se encuentra ante la difícil situación de tener que decidir el inicio de una terapia sustitutiva.

Según las recomendaciones de las guías internacionales estos pacientes deben de cursar con una preparación educativa que genere el conocimiento de la enfermedad renal, las opciones de tratamiento y los posibles cambios en el estilo de vida a fin de constituirse en un apoyo para la toma de decisiones ${ }^{5}$.

Con los resultados obtenidos podemos describir en primer lugar que nuestra población está comprendida en una edad media dominada por los pacientes mayores de 60 años, lo cual constituye el segundo grupo relacionado por el diagnóstico con mayores egresos hospitalarios dentro del IMSS ${ }^{1}$, además de ser a nivel internacional la edad promedio de los pacientes que son admitidos a programas de diálisis; por ejemplo en Japón dos tercios del total de pacientes están por encima de los 60 años y la mitad son mayores de 65 años $^{7}$.

Respecto al estado civil el $73.8 \%$ son casados lo cual nos hace ver la presencia de un cuidador (conyugue, hijos), esta figura es fundamental para levar a cabo el tratamiento sustitutivo renal ya que este grupo de pacientes suele ser parcial o totalmente dependiente ${ }^{8}$.

En relación a la situación clínica de nuestro grupo de estudio, podemos destacar, la presencia de comorbilidad cardiovascular en un $69.2 \%$ de pacientes lo cual constituye la causa más importante de morbimortalidad en los pacientes con IRC y que suele estar presente mucho antes de iniciar el tratamiento diálitico ${ }^{9}$ y que potencialmente podría ser solucionada al iniciar oportunamente la terapia sustitutiva².

En relación al conocimiento de las cuatro dimensiones medidas por el cuestionario se logro aumentar en un $75.4 \%$ el nivel bueno (más de 21 ítems contestados correctamente) cambios que concuerdan con lo publicado en el conocimiento de la enfermedad de los pacientes diabéticos ${ }^{10}$. La 
media en la diferencia de las pruebas pre-post fue de 35.08 comprobando los resultados con prueba $\mathrm{t}$ de Student en 13.207 ( $\mathrm{ns} .000$ ) lo cual respalda nuestra hipótesis.

Al análisis de cada una de las dimensiones de conocimiento integradas en la intervención educativa destaca el "Estilo de Vida" con el mayor aprovechamiento (47.6) encontrando cambios porcentuales hasta de un $42 \%$ en respuestas correctas a preguntas relacionadas con la actividad diaria y la reintegración social al momento de iniciar la terapia de reemplazo.

La segunda dimensión con mayores cambios en el promedio fue "Diálisis" destacando principalmente que el $43 \%$ de los pacientes no consideraban a la diálisis como una opción de tratamiento. El $41 \%$ cambio su perspectiva al considerar que la diálisis es fácil de aprender y se puede realizar en su casa y que al iniciar el tratamiento mejorara su condición de salud.

Cabe destacar que los cambios en el promedio de la dimensión "Función del riñón y conocimiento de la enfermedad renal son significativos, ya que después de la intervención el 99\% de los pacientes logra identificar que se habla de insuficiencia renal cuando el filtrado glomerular cae por debajo de $15 \mathrm{ml} / \mathrm{min}$, así como las funciones básicas del riñón.

Con respecto al inicio oportuno de la terapia sustitutiva se le pregunto a cada paciente del estudio si desea iniciar diálisis en el momento del post test a lo que el $98.5 \%$ respondió que sí. Lo cual nos pone en situación de iniciar los protocolos establecidos para el ingreso a los programas de diálisis.

\section{Conclusiones}

La educación del paciente durante el avance de la enfermedad renal ha demostrado tener impacto sobre los resultados del tratamiento y el planteamiento del tratamiento sustitutivo favorece el inicio de la diálisis en un tiempo apropiado.

Proporcionar información y educación sobre el padecimiento de la insuficiencia renal, a través de metodologías de educación adecuadas sobre los temas ya descritos, incrementa el conocimiento de la enfermedad renal y sus opciones de tratamiento y pudiera asociarse a la toma oportuna de decisiones sobre el inicio oportuno de la terapia sustitutiva.

\section{Agradecimientos}

Agradecemos a los Nefrólogos del Hospital general de Zona No 17 del IMSS que nos han ayudado a la realización de este trabajo y a la MCE María de los Angeles Marroquín Cardona por su asesoría metodológica.

Recibido: Septiembre 2011

Revisado: Septiembre 2011

Modificado: Septiembre 2011

Aceptado: Octubre 2011

\section{Bibliografía}

1. Zambrano Marcela. Zurita Beatriz, Ramírez Teresita de Jesús. Gasto hospitalario de cinco patologías de alto impacto económico, Revista Médica del IMSS 2008; 461: 43-50.

2. Gorriz J. L., Sancho A., Tallardo L.M., Significado pronóstico de la diálisis programada en pacientes que inician tratamiento sustitutivo renal, un estudio Multicentrico español, Nefrología Vol. XXII. Núm. 12002.

3. Recomendaciones de la Sociedad Española de Enfermería Nefrológica (SEDEN) respecto a la creación de consultas de enfermería nefrológica y dotación de las unidades de diálisis peritoneal con recursos de enfermería, 2002. Consultado en http://www.seden. org/files/Documentos\%20para\%20SEDEN\%20 sobre\%20grupo\%20apoyo\%20DP.pdf.

4. Programa de Inicio planeado, un inicio planeado mejora la calidad de vida del paciente, Baxter México, 2006.

5. The National Kidney Foundation Kidney Disease Outcomes Quality Initiative (NKF KDOQI www.kidney.org , Professionals , KDOQI. 
6. C. Santillan Torres. Diseño, implementación y evaluación de un programa educativo para pacientes con IRC. Facultad de Psicología UNAM. Revista Latinoaméricana de Medicina Conductual, Vol. 1 Num. 18 - 201000 37-46.

7. Kurokawa K, Nangaku M, Saito A, et al. Current issues and future perspectives of chronic renal failure. J Am Soc Nephrol 2002; 13: 53-56.

8. J. Teixedo, L. Tarrats, N Arias. Cuestionario de sobrecarga de cuidadores de pacientes de diálisis peritoneal- Nefrología vol. 26 Núm. 12006.
9. L. Rodríguez, F. Ariel, A Serrano et al. Factores de riesgo y complicaciones cardiovasculares en los pacientes bajo diálisis peritoneal. Revista Cubana de Medicina Intensiva y Emergencias 2005;4 (1).

10. T. Jordán Severo. Evaluación del efecto de una intervención educativa en los pacientes diabéticos de cuatro consultorios. Revista Cubana de Med. Gen. Integr. 2007, 23 (2). 